\title{
Musgos de Zacatecas, México III. Síntesis y fitogeografía
}

\author{
Claudio Delgadillo M. y Ángeles Cárdenas S. ${ }^{1}$
}

RESUMEN. En esta contribución se enumeran 37 taxa de musgos nuevos para la flora del estado de Zacatecas. El listado florístico revisado indica que la flora conocida de esa entidad consta de 115 especies y variedades de musgos; no se han incluido el género Atrichum y una especie desconocida de Grimmia. Por su distribución en el mundo estos taxa pueden agruparse en cuatro elementos: Amplia Distribución, Mexicano, Mesoamericano y Endémico. Por su distribución en México, $57 \%$ de la flora se conoce prácticamente de todos los estados de la República Mexicana, mientras que el remanente se distribuye del Eje Neovolcánico hacia el norte. La distribución en Zacatecas apoya la división del estado en dos provincias fitogeográficas: Sierra Madre Occidental y Altiplanicie. Tomando en cuenta aspectos de la geología histórica de Zacatecas y en ausencia de fósiles, se sugiere que esta flora se originó en el Terciario.

ABSTRACT. This contribution lists 37 new moss taxa from the State of Zacatecas, Mexico. The revised listing includes 115 species and varieties of mosses but unidentified specimens of Atrichum and Grimmia are not yet included. On account of their world distribution the taxa can be grouped in four elements: Wide Distribution, Mexican, MesoAmerican and Endemic. Fifty-seven per cent of this moss flora is widely distributed in Mexico while the remainder is known only from the Neovolcanic Belt northwards. Moss distribution in Zacatecas supports the state subdivision into two phytogeographical provinces: Sierra Madre Occidental and Altiplanicie. This moss flora seems to have originated in Tertiary times.

En 1978 se iniciaron los trabajos de campo en el estado de Zacatecas para conocer los musgos de su flora, contribuir con datos florísticos para el manual de musgos mexicanos propuesto por A.J. Sharp y determinar el significado fitogeográfico del Eje Neovolcánico. En nuestra primera contribución presentamos un listado de 29 especies y variedades nuevas para el estado (Delgadillo y Cárdenas, 1979); en la segunda, después de visitar otros valles y algunas elevaciones, publicamus una lista con 47 nuevos taxa para Zacatecas que incluía varias correcciones al listado original (Cárdenas y Delgadillo, 1984).

${ }^{1}$ Departamento de Botánica, Instituto de Biología, Universidad Nacional Autónoma de México, Apdo. Postal 70-233, 04510 México, D.F. 
Debido a que los trabajos de campo prosiguieron en 1983 en el sur y suroeste del estado, se ha hecho necesario presentar una última contribución que incluya los taxa no registrados y un análisis de las relaciones fitogeográficas de toda la flora de musgos.

\section{ADICIONES A LA FLORA}

Los ejemplares colectados en 1983 también se citan con la numeración de uno de nosotros (Cárdenas) después del nombre de cada taxon y de su microhabitat. Todos los ejemplares están depositados en el Herbario Nacional de México (MEXU). Las localidades, fechas, número de colecta y habitat de estas colecciones se enumeran a continuación; los números que no aparecen aquí se incluyeron en las publicaciones previas.

Agosto 26, $198311 \mathrm{~km} \mathrm{NNW}$ de San Pedro Piedra Gorda. $22^{\circ} 31^{\prime}$ N $102^{\circ} 23^{\prime}$ W. Alt. $2080 \mathrm{~m}$.

2999-3006 Matorral, cerca de zona de cultivo con Opuntia, Jatropha, Dodonaea, Mimosa $\mathrm{y}$ otras.

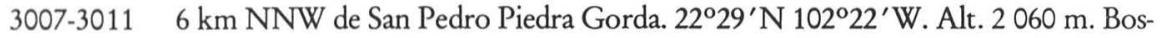
que muy abierto de Juniperus con Opuntia.

3012-3013 4 km NNW de San Pedro Piedra Gorda. $22^{\circ} 29^{\prime} \mathrm{N} 102^{\circ} 22^{\prime}$ W. Alt. 2090 m. Bosque de Juniperus muy abierto con gramíneas muy pequeñas formando un césped, Asclepias, Opuntia y otras.

Agosto 27, $19837 \mathrm{~km} \mathrm{~S}$ de la Laguna Grande, al N de Monte Escobedo. $22^{\circ} 27^{\prime} \mathrm{N} 103^{\circ} 33^{\prime} \mathrm{W}$.

3014-3063 Alt. $2030 \mathrm{~m}$. Bosque de Quercus en fondo de cañada con arroyo y abundantes afloramientos rocosos.

3064-3070 $8 \mathrm{~km} \mathrm{E}$ de Monte Escobedo. $22^{\circ} 21^{\prime} \mathrm{N} 103^{\circ} 30^{\prime} \mathrm{W}$. Alt. $2030 \mathrm{~m}$. Bosque de Quercus sobre suelos someros muy pedregosos.

Agosto 28, 1983 Jerez de García Salinas. $22^{\circ} 38^{\prime} \mathrm{N} 103^{\circ} 00^{\prime} \mathrm{W}$. Alt. $2026 \mathrm{~m}$.

3071-3072

3073-3084 Ruinas de Chicomostoc. $22^{\circ} 27^{\prime} \mathrm{N} 102^{\circ} 48^{\prime} \mathrm{W}$. Alt. $\pm 2100 \mathrm{~m}$.

Agosto $29,19831 \mathrm{~km} \mathrm{~S}$ de Troncoso. $22^{\circ} 44^{\prime} \mathrm{N} 102^{\circ} 21^{\prime}$ W. Alt. $2180 \mathrm{~m}$. Matorral de Opuntia 3085-3094

3095-3099 $1 \mathrm{~km} \mathrm{~W}$ de Providencia. $22^{\circ} 36^{\prime} \mathrm{N} 102^{\circ} 10^{\prime} \mathrm{W}$. Alt. $2250 \mathrm{~m}$. Matorral con Opun tia y Yucca, Ferocactus, Mammillaria y otras.

Amblystegium serpens (Hedw.) B.S.G. Roca, en sitios sombreados. 1160, 1161. 1158 .

Anomobryum filiforme var. concinatum (Spruce) Boul. Suelo, en sitios soleados. 1085,

Atrichum sp. Suelo, en sitios sombreados. 861. Las revisiones más recientes del género (Ireland, 1969; Nylhom, 1971) no son satisfactorias para la identificación de nuestros taxa. Por ello aquí sólo registramos la existencia del género en Zacatecas.

Barbula convoluta Hedw. Suelo cubriendo rocas, en sitios sombreados. 3046.

Bartramia microstoma Mitt. Suelo cubriendo roca, en sitios sombreados. 859c.

Brachymenium mexicanum Mont. Suelo o roca, en sitios expuestos o sombreados. 774, 877, 1088b, 3027. 
B. niveum Besch. Rama o tronco de Quercus, en sitios sombreados. 788, 790, 817, 838. Bryoerythrophyllum inaequalifolium (Tayl.) Zand. Suelo cubriendo roca, en sitios expuestos. 756, 757a.

B. recurvirostrum (Hedw.) Chen var. recurvirostrum. Suelo, en sitios sombreados. 862.

Bryum billardieri Schwaegr. Suelo o roca, en sitios sombreados, soleados o expuestos. 848,868 b, $1076 \mathrm{a}$.

B. robustum Hampe. Suelo en base de helecho, en sitios sombreados. 1143, 1154, 1188.

Campylopus chrismarii (C.M.) Mitt. Suelo en grieta de roca, en sitios sombreados. 734, 735, 3047, 3065.

C. fragilis (Brid.) B.S.G. Suelo cubriendo roca, en sitios sombreados. 750.

C. oblongus Thér. Suelo en grieta de roca, en sitios sombreados o soleados. 844,855 , $860,869 \mathrm{c}$.

C. tallulensis Sull. \& Lesq. Suelo cubriendo roca en sitios sombreados. $749 \mathrm{~b}$.

Curviramea mexicana (Thér.) Crum. Suelo cubriendo rocas, en sitios sombreados o expuestos. 3056a, 3058, 3059a, 3063. Previamente conocido como Pilotrichum mexicanum (Crum 1984).

Didymodon rigidulus Hedw. var. subulatus (Thér. \& Bartr.) Zand. Suelo cubriendo roca, en sitios expuestos. 1065, 1066a, 1169.

Entosthodon longicollis Mitt. Suelo cubriendo roca, en sitios sombreados. 3019, 3020.

Eurbynchium riparioides (Hedw.) Rich. Suelo cubriendo rocas, en sitios sombreados o expuestos. 1109, 3057a, 3063a.

Fissidens fontanus (B.-Pyl.) Steud. Roca sumergida, en sitios sombreados. 3044.

F. weirii Mitt. var. hemicraspedophyllus (Card.) Pursell. Suelo, en sitios sombreados. 3031.

Funaria bygrometrica Hedw. var. calvescens (Schwaegr.) Mont. Suelo cubriendo roca, en sitios expuestos. $320 \mathrm{~b}$.

Grimmia apocarpa Hedw. Suelo cubriendo roca, en sitios sombreados. 813.

G. arizonae Ren. \& Card. Roca o suelo cubriendo roca, en sitios expuestos, soleados o sombreados. 781, 832, 1097, 3048, 3094. El ejemplar 421 registrado como $G$. affinis (Delgadillo y Cárdenas, 1979) pertenece a este taxon.

G. pilifera P. Beauv. Suelo cubriendo roca, en sitios expuestos. 343.

Grimmia sp. Suelo o roca, en sitios expuestos o soleados. 736, 752, 3092. Los ejemplares citados presentan características muy particulares que no coinciden con las de ninguna de las especies conocidas para México. Nuestros ejemplares presentan hojas no quilladas, lámina foliar biestratificada, costa sin células guía y pelo hialino casi liso; las hojas del periquecio cerca de la cápsula son ovado-lanceoladas, envainantes con los bordes y parte media de la lámina diferenciados con células hialinas; la cápsula es inmersa.

Haplocladium microphyllum (Hedw.) Bartr. Roca, en sitios soleados. 794, 809b.

Orthotrichum pycnophyllum Schimp. Rama de Quercus o tronco de Prunus, en sitios sombreados. $791,865,875$

Philonotis fontana. (Hedw.) Brid. Roca, en sitios soleados. 1089.

P. gracillima Ångstr. Suelo cubriendo roca, en sitios soleados. 3040a.

Platygyrium fuscoluteum Card. Base de Quercus, en sitios sombreados. 845.

Ptychomitrium chimborazense (Spruce ex Mitt.) Jaeg. 326, 330a. Originalmente listados como P. leibergii (Delgadillo y Cárdenas, 1979). 
Recopilum tomentosum (Hedw.) Brid. Suelo cubriendo roca, en sitios sombreados. 3056b.

Taxiphyllum taxirameum (Mitt.) Fleisch. Suelo cubriendo roca, en sitios sombreados. 3028, 3057b, 3070.

Tortula amphidiacea (C.M.) Broth. Suelo, en sitios sombreados. 885 .

T. pagorum (Milde) De Not. Tronco de Fraxinus, Opuntia, Salix o Yucca, en sitios sombreados o soleados. 880, 1198a, 1215, 3098.

Weissia andersoniana Zand. Suelo, en sitios sombreados o expuestos. 3051, 3066.

En el listado anterior se enumeran 37 taxa que no fueron citados en las contribuciones precedentes; uno de ellos, $P$. chimborazense, es una corrección al listado de 1979. Debe agregarse Tortula heteroloma Card. que Mishler (en prensa) registró recientemente. En total, la flora conocida de musgos del estado de Zacatecas incluye 115 especies y variedades. Este número es comparativamente pequeño si se considera que la superficie del estado es de más de $75000 \mathrm{~km}^{2}$ y que por su topografía existen ambientes en los que puede desarrollarse una brioflora más diversa. Obviamente, muchas localidades todavía pueden enriquecer el listado florístico y la recolección sucesiva debe hacer énfasis en aquellas que no aparecen en el mapa de nuestras visitas (fig.1); además, deben programarse otras para obtener muestras de los taxa anuales o de vida efímera que hayan faltado en nuestras colecciones. No obstante, debido al predominio de los climas secos (BS) en el estado, la aparente baja humedad relativa de la atmósfera y la alta insolación de los sustratos hacen suponer que el reducido número de especies de musgos es una característica inherente a Zacatecas. Sólo algunos taxa, como la familia Pottiaceae, son particularmente numerosos; ésta incluye 50 especies y variedades, o sea, casi la mitad de la flora. También son importantes las familias Bryaceae (10 spp.), Grimmiaceae (8 spp. incluyendo Ptychomitrium), Bartramiaceae, Dicranaceae y Fissidentaceae (con cinco especies cada una). En conjunto, las seis familias representan más del $70 \%$ de la flora conocida de musgos.

\section{DistribUCIÓN}

Varios autores han discutido la flora y vegetación del estado de Zacatecas en conexión con sus investigaciones sobre el desierto chihuahuense. Otros como Gentry (1957), Guzmán y Vela $(1960)$ y Rzedowski $(1957,1968,1973)$ han hecho alusión directa o indirecta a los tipos de vegetación después de estudiar ciertas áreas del estado o zonas adyacentes al mismo. Debería esperarse que, con estos antecedentes, hubiera un mejor conocimiento de las relaciones fitogeográficas de la flora. Sin embargo, la fitogeografía de Zacatecas sólo se ha abordado de manera muy general en la literatura botánica. Rzedowski (1978), por ejemplo, hace notar que Zacatecas forma parte de dos provincias fitogeográficas: la de la Sierra Madre Occidental y la de la Altiplanicie. En la primera el elemento holártico prevalece ligeramente sobre el neotropical y sobre el autóctono, mientras que en la segunda se puede observar una importante influencia de elementos de la Provincia de la Planicie Costera del Noreste. Recientemente, Acosta (1982) ha hecho notar que los macromicetos del sur de Zacatecas mantienen, como flora, relaciones similares a las que se describen para otras partes del país y que en ese estado algunas 


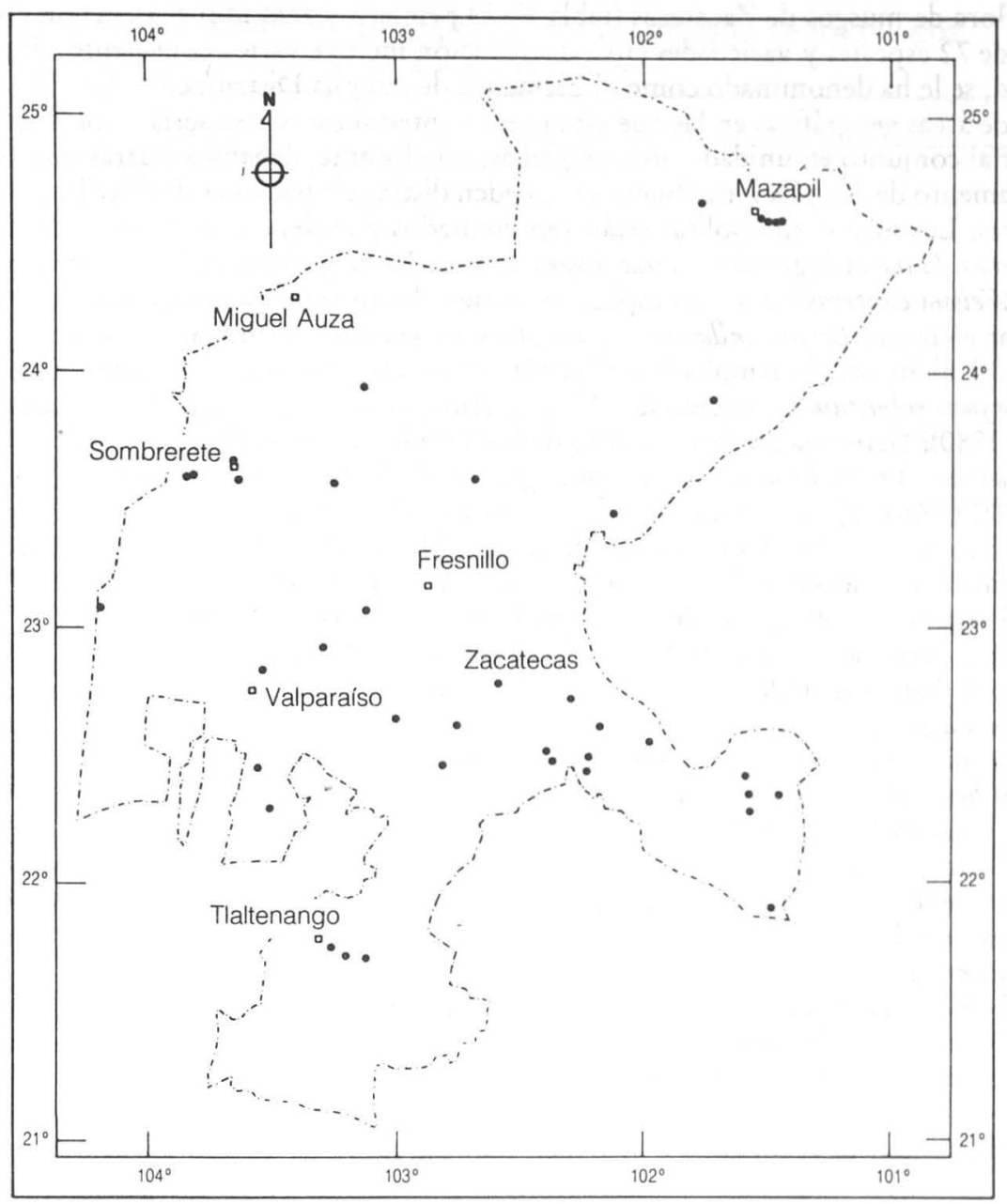

Fig. 1. Localidades de muestreo de musgos en el estado de Zacatecas. Se incluyen las áreas citadas por Delgadillo y Cárdenas (1979); Cárdenas y Delgadillo (1984). Mapa preparado por F. Villegas.

especies no son claramente de distribución holártica o neotropical.

En esta contribución intentamos discutir la distribución de la flora de musgos del estado de Zacatećas, sus relaciones geográficas y su significado en la fitogeografía histórica de México. Al mismo tiempo, deseamos aportar datos para apoyar la delimitación de dos provincias florísticas en esa entidad.

Por su distribución en el mundo se pueden reconocer cuatro grupos fundamentales 
en la flora de musgos de Zacatecas (tabla 1). El primero y más numeroso es un contingente de 72 especies y variedades cuya distribución incluye varios continentes. Por este motivo, se le ha denominado como el Elemento de Amplia Distribución. Debido al número de áreas geográficas en las que están representados estos taxa sería impráctico subdividir al conjunto en unidades más pequeñas; no obstante, debemos aclarar que dentro del Elemento de Amplia Distribución se pueden distinguir patrones de distribución particulares. Los taxa cosmopolitas están representados por Bryum argenteum, Ceratodon purpureus, Funaria bygrometrica var. hygrometrica, Hedwigia ciliata, Leptobryum pyriforme y Weissia controversa. Otras especies y variedades tienen una distribución pantropical, por ejemplo, Bryum billardieri y Fissidens asplenioides. También se incluyen como parte del Elemento de Amplia Distribución varios taxa que son claramente disyuntos, e.g., Bryum robustum (conocido de México, Madagascar, Australia y Nueva Caledonia; Ochi, 1980), Grimmia pilifera (del este de los Estados Unidos, México y Japón; Crum y Anderson, 1981), Leptodontium capituligerum (de México-Sudamérica y Affrica; Zander, 1972), Racopilum tomentosum (de América y África) y Tortula chisosa (del suroeste de los Estados Unidos, México y sur de África; Magill, et al., 1983). La mayoría de los taxa remanentes tienen su distribución principal en los continentes del Hemisferio Norte.

El segundo contingente, denominado Elemento Mexicano, está constituido por 20 especies y variedades y se distribuye del suroeste de los Estados Unidos hasta Guatemala; sólo se han registrado tres especies de El Salvador, Costa Rica, Panamá, Jamaica o las Islas Vírgenes. Varios taxa como Didymodon rigidulus var. subulatus, Funaria apiculatopilosa, Grimmia arizonae, Homomalium mexicanum, Phascum byalinotrichum, Platygyrium fuscoluteum, Tortula aurea y Weissia andersoniana sólo se conocen del suroeste de los Estados Unidos y México.

El tercer grupo, el Elemento Mesoamericano, se distribuye esencialmente de México al norte de Sudamérica; varios taxa de este elemento pueden tener áreas de distribución más amplia hacia el norte o sur, pero de las 18 especies y variedades solamente tres se conocen de las Antillas.

El último grupo, el Elemento Endémico, está compuesto por cinco taxa conocidos exclusivamente de la República Mexicana.

Con el propósito de determinar si la distribución de los musgos de Zacatecas apoya la idea de que su territorio está comprendido dentro de dos provincias fitogeográficas o geológicas (cf. Rzedowski, 1978; López Ramos, 1979), se dividió arbitrariamente el estado en franjas longitudinales que corresponden a los principales meridianos que lo cruzan. En la tabla 1 se muestran tales divisiones y se indican los taxa que se han observado en ellas. Es evidente, a partir de estos datos, que existen muchos taxa que están representados desde el este hasta el oeste de Zacatecas. Otros sólo se han registrado de las zonas orientales u occidentales, pero no de localidades intermedias; es probable que éstos, si no ocupan habitats especializados, también tengan una distribución generalizada en el estado. Además de estos taxa, en la tabla 1 se muestra que existen otros que están restringidos a ciertas franjas longitudinales. En el extremo oriental hay 17 especies $\mathrm{y}$ variedades que no se han registrado de otras zonas; entre ellos, Aloina rigida var. rigida, Amblystegium serpens, $A$. varium y Encalypta vulgaris se conocen únicamente de localidades al oeste de Concepción del Oro. En la zona central hay 11 especies y variedades conocidas exclusivamente de ella pero potencialmente la mayoría debe encontrarse también en el sector occidental. De este último se conocen 44 taxa que no observamos 
TABLA 1. Elementos fitogeográficos y distribución de los musgos en Zacatecas, México.

Elemento/taxa

AMPLIA DISTRIBUCIÓN

Aloina bifrons

A. rigida var. rigida

Amblystegium serpens

A. varium

Anacolia laevisphaera

Anoectangium aestivum

Anomobryum filiforme var. filiforme

A. $f$. var. concinatum

Barbula convoluta

Braunia secunda

Bryoerythrophyllum inaequalifolium

B. jamesonii

B. recurvirostrum var. recurvirostrum

Bryum argenteum

B. billardieri

B. robustum

Campylium hispidulum var. sommerfeltii

Campylopus fragilis

C. pilifer

Ceratodon purpureus

Crossidium crassinerve var. crassinerve

Desmatodon convolutus

Didymodon australasiae var. australasiae

D. a. var. umbrosus

D. rigidulus var. gracilis

D. r. var. icmadophila

D. r. var. rigidulus

D. tophaceus

D. vinealis var. luridus

D. v. var. vinealis

Encalypta vulgaris

Eurbynchium riparioides

Fabronia ciliaris

F. pusila

Fissidens asplenioides

F. fontanus

F. milobakeri

Funaria bygrometrica var. bygrometrica

F. h. var. calvescens

Grimmia affinis

G. apocarpa

G. ovalis

G. pilifera

Haplocladium microphyllum

Hedwigia ciliata

Hyophila involuta

Leptobryum pyriforme

Leptodontium capituligerum

L. flexifolium

Lindbergia brachyptera
1

2

3

$X$
$X$
$X$
$X$

$\mathrm{X}$

$\mathrm{X}$

$\mathrm{X}$

$\mathrm{X}$

$\mathrm{X}$

$\mathrm{X}$
$\mathrm{X}$
$\mathrm{X}$
$\mathrm{X}$
$\mathrm{X}$
$\mathrm{X}$
$\mathrm{X}$
$\mathrm{X}$
$\mathrm{X}$
$\mathrm{X}$
$\mathrm{X}$

N

$\mathrm{X}$

$\mathrm{X}$

N

N

N

N

N

$\mathrm{N}$

N

$\mathrm{N}$

$\mathrm{X}$

X

$\mathrm{X}$

$\mathrm{X}$

$\mathrm{X}$

$\mathrm{X}$

$\mathrm{X}$

$\mathrm{X}$

$X$

$\mathrm{X} \quad \mathrm{X}$

$\mathrm{X}$

$\mathrm{X}$

$\mathrm{N}$

N

$\mathrm{N}$

$\mathrm{X}$

$\mathrm{X}$

$\mathrm{X}$

X

N

$\mathrm{X}$
N
N

$\mathrm{N}$

$\mathrm{N}$

T. 


\begin{tabular}{|c|c|c|c|c|}
\hline Elemento/taxa & 1 & 2 & 3 & \\
\hline Molendoa sendtneriana & $\mathrm{X}$ & & & \\
\hline Orthotrichum diaphanum & $\mathrm{X}$ & $\mathrm{X}$ & & $\mathrm{N}$ \\
\hline Oxystegus tenuirostris & $\mathrm{X}$ & & $\mathrm{X}$ & \\
\hline Phascum cuspidatum & $\mathrm{X}$ & $\mathrm{X}$ & $\mathrm{X}$ & $\mathrm{N}$ \\
\hline Philonotis fontana & & & $\mathrm{X}$ & $\mathrm{N}$ \\
\hline P. marchica & $\mathrm{X}$ & & & $\mathrm{N}$ \\
\hline Pleuridium subulatum & & & $\mathrm{X}$ & $\mathrm{N}$ \\
\hline Poblia elongata & $\mathrm{X}$ & & $\mathrm{X}$ & \\
\hline Pterygoneurum ovatum & $\mathrm{X}$ & & & $\mathrm{N}$ \\
\hline P. subsessile & & $\mathrm{X}$ & & $\mathrm{N}$ \\
\hline Racopilum tomentosum & & & $\mathrm{X}$ & \\
\hline Taxiphyllum taxirameum & & & $\mathrm{X}$ & \\
\hline Timmiella anomala & & & $\mathrm{X}$ & \\
\hline Tortula chisosa & $\mathrm{X}$ & $\mathrm{X}$ & $\mathrm{X}$ & $\mathrm{N}$ \\
\hline T. fragilis & $\mathrm{X}$ & $\mathrm{X}$ & $\mathrm{X}$ & \\
\hline T. pagorum & $\mathrm{X}$ & $\mathrm{X}$ & & $\mathrm{N}$ \\
\hline T. papillosa & $\mathrm{X}$ & & & N \\
\hline Trichostomum brachydontium & $\mathrm{X}$ & $\mathrm{X}$ & $\mathrm{X}$ & \\
\hline T. crispulum & $\mathrm{X}$ & & $\mathrm{X}$ & \\
\hline Weissia condensa & $\mathrm{X}$ & & $\mathrm{X}$ & $\mathrm{N}$ \\
\hline W. controversa & $\mathrm{X}$ & & & \\
\hline Zygodon viridissimus & & & $\mathrm{X}$ & \\
\hline \multicolumn{5}{|l|}{ MEXICANO } \\
\hline Aloina bamulus & $\mathrm{X}$ & $\mathrm{X}$ & $\mathrm{X}$ & \\
\hline Bartramia microstoma & & & $\mathrm{X}$ & \\
\hline Brachymenium mexicanum & & $\mathrm{X}$ & $\mathrm{X}$ & \\
\hline Bryoerythrophyllum recurvirostrum var. aeneum & $\mathrm{X}$ & & & \\
\hline Didymodon rigidulus var. subulatus & & & $X$ & $\mathrm{~N}$ \\
\hline Entosthodon longicollis & & & $\mathrm{X}$ & \\
\hline Fabronia wrightii & $\mathrm{X}$ & $\mathrm{X}$ & $\mathrm{X}$ & \\
\hline Funaria apiculatopilosa & $\mathrm{X}$ & $\mathrm{X}$ & $\mathrm{X}$ & $\mathrm{N}$ \\
\hline Grimmia arizonae & & $\mathrm{X}$ & $\mathrm{X}$ & $\mathrm{N}$ \\
\hline Homomalium mexicanum & & & $\mathrm{X}$ & $\mathrm{N}$ \\
\hline Lindbergia mexicana & $\mathrm{X}$ & & $\mathrm{X}$ & \\
\hline Neobyophila spatbulifolia & & & $\mathrm{X}$ & \\
\hline Orthotrichum pycnophyllum & & & $\mathrm{X}$ & \\
\hline Phascum brittoniae & & $X$ & & $\mathrm{~N}$ \\
\hline P. hyalinotrichum & $\mathrm{X}$ & $\mathrm{X}$ & & $\mathrm{N}$ \\
\hline Platygyriella pringlei & & & $\mathrm{X}$ & \\
\hline Platygyrium fuscoluteum & & & $\mathrm{X}$ & $\mathrm{N}$ \\
\hline Pogonatum leptopelma & & & $\mathrm{X}$ & \\
\hline Tortula aurea & $\mathrm{X}$ & $\mathrm{X}$ & $\mathrm{X}$ & $\mathrm{N}$ \\
\hline Weissia andersoniana & & & $\mathrm{X}$ & $\mathrm{N}$ \\
\hline \multicolumn{5}{|l|}{ MESOAMERICANO } \\
\hline Barbula spiralis & $\mathrm{X}$ & $\mathrm{X}$ & $\mathrm{X}$ & \\
\hline Campylopus chrismarii & & $\mathrm{X}$ & $\mathrm{X}$ & $\mathrm{N}$ \\
\hline C. oblongus & & & $\mathrm{X}$ & \\
\hline C. tallulensis & & $X$ & & \\
\hline Didymodon revolutus & $\mathrm{X}$ & $\mathrm{X}$ & $\mathrm{X}$ & $\mathrm{N}$ \\
\hline Entodon beyrichii & & & $\mathrm{X}$ & \\
\hline
\end{tabular}




\begin{tabular}{|c|c|c|c|c|}
\hline Elemento/taxa & 1 & 2 & 3 & \\
\hline Fissidens repandus & $\mathrm{X}$ & & $\mathrm{X}$ & \\
\hline F. weirii var. bemicraspedophyllus & & & $\mathrm{X}$ & \\
\hline Leskea angustata & $\mathrm{X}$ & $\mathrm{X}$ & $\mathrm{X}$ & $\mathrm{N}$ \\
\hline Philonotis gracillima & & & $\mathrm{X}$ & \\
\hline Platygyriella densa & & & $\mathrm{X}$ & \\
\hline Pogonatum cuspidatum & & & $\mathrm{X}$ & $\mathrm{N}$ \\
\hline Ptychomitrium chimborazense & & $\mathrm{X}$ & & $\mathrm{N}$ \\
\hline Rhexophyllum subnigrum & & $\mathrm{X}$ & $\mathrm{X}$ & $\mathrm{N}$ \\
\hline Tortula amphidiacea & & $\mathrm{X}$ & & \\
\hline T. obtusissima & $\mathrm{X}$ & $\mathrm{X}$ & $\mathrm{X}$ & \\
\hline T. quitoensis & $\mathrm{X}$ & & & $\mathrm{N}$ \\
\hline Trichostomum jamaicense & $\mathrm{X}$ & & & \\
\hline \multicolumn{5}{|l|}{ ENDÉMICO } \\
\hline Brachymenium niveum & & & $\mathrm{X}$ & \\
\hline Curviramea mexicana & & & $\mathrm{X}$ & \\
\hline Jeffueliobryum arsenei & $\mathrm{X}$ & $\mathrm{X}$ & & $\mathrm{N}$ \\
\hline Pleuridium mexicanum & $\mathrm{X}$ & & & $\mathrm{N}$ \\
\hline Tortula beteroloma & & & $\mathrm{X}$ & $\mathrm{N}$ \\
\hline
\end{tabular}

$1=101^{\circ}-101^{\circ} 59^{\prime} \mathrm{N} ; 2=102^{\circ}-102^{\circ} 59^{\prime} \mathrm{N} ; 3=103^{\circ}-104^{\circ} \mathrm{N}+; \mathrm{N}=$ taxa distribuidos desde el Eje Neovolcánico hacia el norte en México (explicación en el texto).

en las otras franjas. No es improbable que en visitas futuras algunas de estas especies y variedades se encuentren hacia el oriente; Anoectangium aestivum, Barbula convoluta y Neobyophila spathulifolia, por ejemplo, están en este caso. Sin embargo, la mayoría de los taxa exclusivos del sector occidental comúnmente se asocian con los climas más húmedos y frescos de las elevaciones superiores y no es probable que se les encuentre fuera de esta zona en Zacatecas. En consecuencia, desde un punto de vista briológico, coincidimos en apoyar la división del estado en dos provincias fitogeográficas (Sierra Madre Occidental y Altiplanicie) aun cuando por nuestro método de muestreo y análisis no podemos precisar los límites geográficos entre ellas.

En México la flora de musgos de Zacatecas presenta dos tipos de distribución. Aproximadamente el $57 \%$ de los taxa son de amplia distribución y como conjunto se les conoce prácticamente de todos los estados de la República; la mayoría son parte del elemento de Amplia Distribución en el mundo, pero también están incluidos taxa de otros elementos. El remanente de la flora se distribuye hasta los estados de Michoacán, México, Morelos, Puebla y Veracruz, pero aparentemente no se extiende hacia el sureste del país. En la tabla 1 los componentes de este grupo se han señalado con una "N" para resaltar su distribución en el norte de México.

\section{ALGUNAS CONSIDERACIONES HISTÓRICAS}

La distribución conocida de la flora de musgos de Zacatecas hace suponer que sus orígenes y edad son diversos. Es difícil la reconstrucción de los eventos que dieron lugar a 
la distribución actual de estos taxa, principalmente porque no se conocen restos fósiles de briofitas en el área. Por esta razón, y basados en evidencias indirectas, a continuación presentamos una discusión hipotética sobre la fitogeografía histórica de Zacatecas.

López Ramos (1979) ha hecho notar que la provincia geológica del Altiplano Mexicano, de la cual Zacatecas es parte, estuvo ocupada por mares que persistieron hasta fines del Cretácico y principios del Terciario. La actividad tectónica del Oligoceno provocó plegamientos y afallamientos hacia el norte y sur del Altiplano y la actividad volcánica hizo que la mayor parte de la provincia se cubriera de lava en el Mioceno tardío y el Plioceno. Por último, la erosión de las rocas produjo grandes depósitos durante el Cuaternario.

En la provincia geológica de la Sierra Madre Occidental, por otro lado, hubo un levantamiento combinado con un plegamiento intenso de rocas paleozoicas y mesozoicas durante la segunda mitad del Cretácico. La actividad volcánica, los plegamientos y la erosión, dieron lugar a una topografía rugosa y la deposición de rocas en cuencas cerradas al oriente de la Sierra Madre Occidental, particularmente en Zacatecas, en el Terciario Medio y Superior.

Los eventos geológicos descritos permiten suponer que la colonización por briofitas en cualquiera de las dos provincias no ocurrió antes del Terciario. En el Terciario inferior el clima se hizo más caliente y permitió que las floras tropicales y subtropicales migraran hacia las zonas polares (Axelrod, 1950). No hay razón para suponer que Zacatecas quedó al margen de estos cambios y puede asumirse que el estado recibió un número considerable de briofitas neotropicales que ocuparon los habitats disponibles en este periodo. Con la elevación de las montañas y la progresiva aridez del Terciario hubo suficiente oportunidad para la diferenciación de una flora desértica que se estableció preferentemente en el lado oriental desde el Oligoceno Medio. A partir del Mioceno, la brioflora de esta parte de Zacatecas debe haber mantenido un contacto estrecho con la de localidades más al norte; el intercambio florístico entre esas áreas se manifiesta actualmente por la presencia de un contingente de especies distribuidas entre el suroeste de los Estados Unidos y Guatemala (Elemento Mexicano) y por aquellas que tienen una distribución preferencial hacia el norte de México (cf. tabla 1). Este mismo hecho explica que la flora de musgos de Zacatecas incluya sólo unos cuantos endemismos.

La tendencia hacia un clima continental más seco que se inició a principios del Terciario tuvo su culminación en el Plioceno Medio. Sin embargo, para el Plioceno Superior el clima se hizo más fresco y más húmedo (Axelrod, 1950). Estos cambios, junto con los que ocurrieron durante el Pleistoceno, deben haber modificado las áreas de distribución de las briofitas de Zacatecas extendiéndolas o reduciéndolas proporcionalmente. No debemos pasar por alto que Meyer (1973) ha mostrado que en el área de Cuatro Ciénegas, Coahuila, el clima del Cuaternario no se modificó en los últimos 30000 a 40000 años. Si este hecho se hace extensivo al resto del desierto chihuahuense, las interpretaciones fitogeográficas pueden modificarse ligeramente pues el periodo involucrado es breve.

Axelrod (1950) sugiere que los taxa emparentados que ahora se encuentran en áreas de desierto ampliamente separadas, derivaron de floras terciarias que anteriormente las conectaban. Estos argumentos también pueden aplicarse a los musgos asociados con esas floras y explican la distribución discontinua de poblaciones de Aloina bifrons, Barbula spiralis y Didymodon revolutus, cuando menos en el continente americano. Las disyun- 
ciones intercontinentales son difíciles de explicar sin asumir que los taxa involucrados son muy antiguos y que forman parte de floras preterciarias que se establecieron en la vecindad de las áreas que actualmente ocupan.

En cuanto a los taxa de la provincia de la Sierra Madre Occidental, que no se han encontrado hacia el oriente de la misma en Zacatecas, podría asumirse que son parte de las floras que migraron en sentido norte-sur después de la formación de dicha cordillera (cf. Delgadillo, 1971) y su historia está íntimamente ligada a la de las elevaciones altas del centro de México. Algunas especies, sin embargo, se encuentran ocasionalmente en el occidente de Zacatecas porque requieren de habitats particulares (e.g. Fissidens fontanus, acuático; Neobyophila spathulifolia, calcífila) y su presencia está más bien ligada a la distribución de estos ambientes.

En resumen, se puede sugerir que la flora de musgos de Zacatecas es el resultado de migraciones de plantas a lo largo del Terciario. Los elementos que la componen indican que sus contactos han sido múltiples pero aún no se pueden determinar los detalles de su evolución y dispersión a través del tiempo. Cualquier inferencia posterior debe provenir de datos paleobotánicos - lo cual es improbable para musgos - o del análisis de las interrelaciones filogenéticas de algunos miembros de la flora.

AGRADECIMIENTOS. Los autores agradecen la verificación o la identificación de ejemplares problemáticos de Fissidens al Dr. Ronald A. Pursell, de la Pennsylvania State University. Al Dr. Frank D. Bowers, de la University of Wisconsin-Stevens Point, que proporcionó información valiosa para la identificación de otras muestras, y por último, al Dr. Jerzy Rzedowski, por la revisión crítica del manuscrito.

\section{LITERATURA CITADA}

Acosta C., S. 1982. Contribución al conocimiento florístico y ecológico de los macromicetos del sur de Zacatecas. Tesis Esc. Nal. Cs. Biol., IPN México, D.F. 92 p.

AXELROD, D.I. 1950. Evolution of desert vegetation in western North America. Carnegie Inst. Washington Publ. 590:215-306.

Cárdenas S., M. A. y C. Delgadillo M. 1984. Musgos de Zacatecas, México. II. Bol. Soc. Bot. México 46:13-18. Crum, H. 1984. Notes on tropical American mosses. Bryologist 87:203-216.

- y L. E. ANDERSON. 1981. Mosses of eastern North America. Columbia University Press, Nueva York, 2 vols.

Delgadillo M., C. 1971. Phytogeographic studies on alpine mosses of Mexico. Bryologist 74:331-346.

- y M. A. Cárdenas S. 1979. Musgos de Zacatecas, México. I. Bol. Soc. Bot. México 38:1-6.

Gentry, H.S. 1957. Los pastizales de Durango. Inst. Mex. Rec. Nat. Renov., México, D.F., 361 p.

GuZmán H., G. y L. Vela G. 1960. Contribución al conocimiento de la vegetación del suroeste del estado de Zacatecas. Bol. Soc. Bot. México 25:46-61.

Ireland, R.R. 1969. Taxonomic studies on the genus Atrichum in North America. Canad. Jour. Bot. 47:353-368.

López R., E. 1979. Geología de México. México, D.F. vol. Il, 454 p.

Magill, R. E., C. Delgadillo M. y L.R. STARK. 1983. Tortula chisosa sp. nov., a bistratose-leaved species from the United States, Mexico, and southern Africa. Ann. Missouri Bot. Gard. 70:200-202.

Meyer, E.R. 1973. Late-Quaternary paleoecology of the Cuatro Ciénegas Basin, Coahuila, Mexico. Ecology 54:982-995.

MishleR, B.D. Tortula. En: A.J. Sharp y H.A. Crum (eds.), Manual of Mexican mosses (en prensa). 
NYHOLm, E. 1971. Studies in the genus Atricbum P. Beauv. A short survey of the genus and the species. Lindbergia 1:1-33.

OCHI, H. 1980. A revision of the Neotropical Bryoideae, Musci (First Part). Jour. Fac. Educ. Tottori Univ., Nat. Sci. 29:49-154.

RzEDOwsKI, J. 1957. Vegetación de las partes áridas de los Estados de San Luis Potosí y Zacatecas. Rev. Soc. Mex. Hist. Nat. 18:49-101.

— 1968. Las principales zonas áridas de México y su vegetación. Bios 1:4-24.

- 1973. Geographical relationships of the flora of Mexican dry regions. En: A. Graham (ed.), Vegetation and vegetational history of northern Latin America. Elsevier Sci. Co., Amsterdam, pp. 61-72. 1978. Vegetación de México. Ed. Limusa. México, D.F., 432 p.

Zander, R.H. 1972. Revision of the genus Leptodontium (Musci) in the New World. Bryologist 75:213-280. 